\title{
The Presence of a Malignant Comorbidity is a Significant Predictor of Increased 30-Day Hospital Readmission Rates in HIV-1 Infected Individuals
}

\section{Juan Carlos Rico', Rebecca M Schwartz ${ }^{2}$, Joseph McGowan ${ }^{1}$ and Daniel O Griffin ${ }^{1,3 *}$}

${ }^{1}$ The Karin and Dayton Brown, Jr. Division of Infectious Diseases, Department of Medicine, North Shore-Long Island Jewish Health System and Hofstra North Shore-LIJ School of Medicine, Manhasset, NY 11030, USA

${ }^{2}$ Department of Occupational Medicine, Epidemiology and Prevention, North Shore-Long Island Jewish Health system and Hofstra North Shore-LIJ School of Medicine, Manhasset, NY 11030, USA

${ }^{3}$ Columbia University Medical Center, Department of Biochemistry and Molecular Biophysics, Department of Medicine-Division of Infectious Diseases, New York, NY 10032, USA

\begin{abstract}
Thirty-day hospital readmission is a benchmark for quality of care. HIV-1-infected individuals have a significantly increased 30-day readmission rate compared to their HIV-1-uninfected peers. Prior work has identified an association between several factors with an increased 30-day readmission rate in HIV-1-infected individuals. With malignancy recognized as a growing problem for HIV-1-infected patients, we sought to determine, in a cohort of 775 hospitalized patients with access to outpatient care, whether the presence of malignancy was a predictor of 30-day readmission. Of these 775 patients, 440 patients met the inclusion criteria for this study. We observed that the presence of a malignant comorbidity was a significant predictor of increased hospital 30-day readmission rates in HIV-1-infected individuals. The odds ratio for 30-day readmission with a co-morbid diagnosis of malignancy was 2.25 with a $95 \%$ confidence interval of $01.24-4.11$ ( $p$-value $=0.008)$.
\end{abstract}

Keywords: HIV-1; Malignancy; Hospital 30-day readmission; Lymphoma; Lung cancer

\section{Introduction}

Investigations have identified 30-day hospital readmission as a frequent and costly problem for Human Immunodeficiency Virus type 1 (HIV-1) infected patients [1]. Thirty-day readmission rate for HIV1 -infected individuals is elevated when compared to HIV-1-uninfected individuals $[2,3]$. Low CD4-count, long hospital stay, homelessness, low socio-economic status, lack of a partner, distance from the hospital, crack cocaine use, initiation of high active antiretroviral therapy (HAART) and non-compliance with HAART are associated with increased 30-day readmission rates in HIV-1-infected patients [3-7]. We evaluated comorbidities for association with 30-day readmission rates. The prevalence of malignancy in the HIV-1-infected population is significant and might impact readmission rates [8-11].

\section{Method}

We performed a retrospective cohort study. Patients eligible for inclusion were HIV-1-infected patients followed at the North Shore-LIJ Health System (NSLIJHS) HIV clinic, just outside the New York Metropolitan area, who were hospitalized at either of the two NSLIJHS teaching hospitals during the period from January 1, 2011 to December 31, 2012. Admissions for elective procedures were excluded. Patients not followed at the NSLIJHS HIV clinic were excluded. Index hospitalization was defined as the first admission during the study period with no admission in the previous 6-months. A 30-day hospital readmission was defined as a non-elective hospitalization that occurred during the next 30-days following discharge from the index hospitalization. Same day readmissions were included among the 30-day readmissions. Index admission and readmission diagnosis were categorized into sixteen categories based on the International Classification of Diseases diagnostic tool version 9 (ICD-9); immune reconstitution inflammatory syndrome/opportunistic infection(IRIS/ OI), newly diagnosed HIV, advanced acquired immune deficiency
syndrome(AIDS), non-AIDS infection, malignancy, hematology, neurology, cardiovascular, pulmonary, gastrointestinal(GI), renal, surgery, psychiatry, obstetrics-gynecology(OB-GYN), musculoskeletal, and endocrine. The presence of the comorbidities diabetes type II(DM-II), hypertension(HTN), chronic obstructive pulmonary disease (COPD), end stage renal disease(ESRD), hepatitis $\mathrm{C}(\mathrm{HCV})$, malignancy, and psychiatric diagnosis were determined. Each categorization was based on review of the medical record. Patient information was obtained from review of the NSLIJHS medical record. Univariate associations were determined using Chi Square tests for categorical variables and Analysis of Variance for continuous variables. Multivariable analysis utilized logistic regression. Significance was set at $\mathrm{p}<0.05$ and $95 \%$ Confidence Intervals (CI). Statistical analysis was performed using SPSS (version 21) statistical software. All research was conducted in accordance with the standards of and with the approval of the NSLIJHS IRB.

\section{Results}

During the 2-years from January 1, 2011 to December 31, 2012, 775 HIV-1-infected patients were hospitalized at the two NSLIJHS teaching hospitals. Of these 775 patients, 440 patients met the inclusion criteria.

*Corresponding author: Daniel O Griffin, Department of Biochemistry and Molecular Biophysics, Columbia University Medical Center, HHSC 1310c, 701 West 168th Street, New York, NY 10032, USA, Tel: 212-305-3794; Fax: 212-3055106; E-mail: dg2810@columbia.edu

Received November 17, 2015; Accepted December 20, 2015; Published December 26, 2015

Citation: Rico JC, Schwartz RM, McGowan J, Griffin DO (2015) The Presence of a Malignant Comorbidity is a Significant Predictor of Increased 30-Day Hospital Readmission Rates in HIV-1 Infected Individuals. J AIDS Clin Res 6: 532 doi:10.4172/2155-6113.1000532

Copyright: ( 2015 Rico JC, et al. This is an open-access article distributed under the terms of the Creative Commons Attribution License, which permits unrestricted use, distribution, and reproduction in any medium, provided the original author and source are credited. 
The mean age of patients was 48.6 years (range=18-84). Patients were $43.9 \%$ female $(n=193)$ and $56.1 \%$ male $(n=247)$. Mean length of stay was $9.5+/-10.2$ days (mean+/-SD). Thirty-day readmission rate for all patients was $23.2 \%$.

Index admission diagnoses were distributed among various categories with non-AIDS infections being the largest single category accounting for over $20 \%$ of index admission diagnoses. (Figure 1a) Only $6.1 \%$ of index admissions were classified as due to a diagnosis of malignancy. (Figure 1a) Non-AIDS infections were also the most common principle diagnosis for readmission but malignancy was the second most common categorization for readmission, having nearly tripled relative to index admission rates (Figure $1 \mathrm{~b}$ ).

Opportunistic infections (OIs) included mycobacterium avium intracellulare (MAI), toxoplasmosis, oral candidiasis, progressive multifocal encephalopathy (PML), cryptococcal meningitis, and pneumocystis jirovecii pneumonia. NonAIDS infections included cellulitis, fever, influenza, meningitis (nonAIDS defining), bacteremia, abscess, pyleonephtitis, urinary tract infections, neurosyphilis, wound infections, neutropenic fever, infectious diarrhea, herpes zoster, endocarditis, and pneumonia. Malignancies included lymphoma, lung cancer, Kaposi's sarcoma (KS), breast cancer, multiple myeloma (MM), laryngeal cancer, germ cell tumor, liver cancer, squamous cell cancer (SCC), colon cancer, anal cancer, melanoma, and Castleman's disease. Hematological diagnoses were deep vein thrombosis (DVT), anemia, epistaxis, and other coagulopathies. Neurological issues were acute mental status changes, primary headache (secondary causes excluded), head contusion, neuropathy, cerebral vascular accident (CVA), visual disturbance, dizziness, seizure, and syncope. Cardiovascular diagnoses were cardiac tamponade, post myocardial infarction syndrome, chest pain, congestive heart failure, pericardial effusion, aortic stenosis, HTN, constrictive pericarditis, and arrhythmia. Pulmonary diagnoses were COPD, hemoptysis, dyspnea, and asthma exacerbation. GI issues were small bowel obstruction, abdominal pain, constipation, decompensated liver disease, diarrhea, gastritis, gastrointestinal bleed, ileitis, pancreatitis, nausea with vomiting and ulcerative colitis. Renal diagnoses were nephrolithiasis and acute renal failure. Surgical diagnoses included fistula placement for hemodialysis, thrombectomy, acute abdomen, acute stabbing, abdominal aortic aneurysm, lymph node dissection, coronary artery bypass graft (CABG), hernia repair, and pacemaker placement. Psychiatric conditions were depression, schizophrenia, conversion disorder, bipolar disorder, alcohol intoxication, reactive psychosis, suicidal ideation, anxiety, and drug overdose. OB-GYN conditions were vaginal bleeding, labor, pelvic pain, pregnancy complications, uterine leiomyoma, and benign ovarian neoplasm. The musculoskeletal conditions were calcaneus fracture, hip fracture, femur fracture, back pain, limb pain, herniated disc, limb swelling, avascular necrosis, ankle contusion, and femur fracture. Endocrine issues were DM-II, hypoglycemia, hypokalemia, and hypocalcemia.

Comorbidities present in patients admitted for an index hospitalization were DM-II in $14.5 \%(\mathrm{n}=64), \mathrm{HTN}$ in $34.1 \%(\mathrm{n}=150)$, COPD in $10.2 \%(n=45)$, ESRD in $6.8 \%(n=30)$, HCV in $20 \%(n=80)$, malignancy in $19.5 \%(\mathrm{n}=86)$ and psychiatric diagnosis in $30.9 \%$ $(\mathrm{n}=136)$. HIV-1-infected patients that were readmitted within 30 days of discharge after the index admission were characterized by

\section{A}

Index admission primary diagnosis

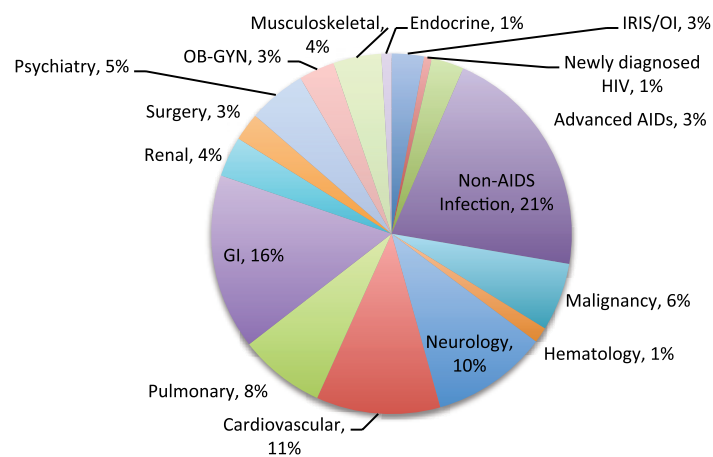

B

\section{Readmission primary diagnosis}

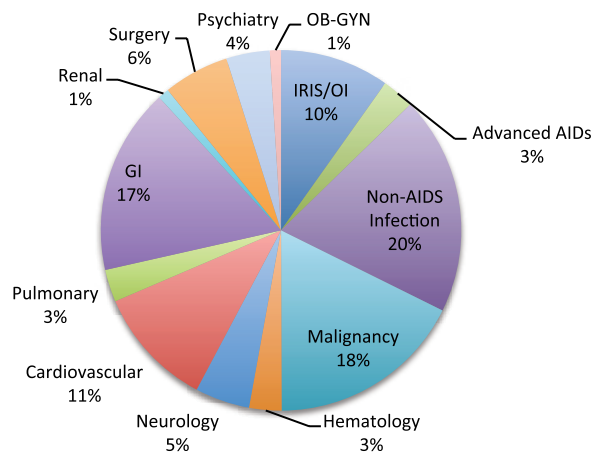

C

\begin{tabular}{|c|c|c|}
\hline Comorbidity & Index admissions \%(n) & Re-admissions \%(n) \\
\hline DM-II & $14.5 \%(64)$ & $11.8 \%(12)$ \\
\hline HTN & $34.1 \%(150)$ & $38.2 \%(39)$ \\
\hline COPD & $10.2 \%(45)$ & $12.7 \%(13)$ \\
\hline ESRD & $6.8 \%(30)$ & $9.8 \%(10)$ \\
\hline HCV & $20 \%(80)$ & $18.6 \%(19)$ \\
\hline Malignancy & $\mathbf{1 9 . 5 \% ( 8 6 )}$ & $\mathbf{3 0 . 4 \% ( 3 1 )}$ \\
\hline Psychiatric Diagnosis & $30.9 \%(136)$ & $36.3 \%(37)$ \\
\hline
\end{tabular}

Figure 1: Index and 30-day readmission diagnoses and comorbidities. (A) Percentages of each diagnosis category are shown for index admissions. (B) Percentages of each primary diagnosis category are shown for 30-day readmissions. (C) Table showing the percentage of patients with index admissions and 30-day readmissions with particular comorbidities.

IRIS (Immune Reconstitution Inflammatory Syndrome), OI (Opportunistic Infection) AIDs (Acquired Immune Deficiency Syndrome), GI (Gastrointestinal), OB-GYN (Obstetrics and Gynecology), DM-II (Diabetes Mellitus type II), HTN (Hypertension), COPD (Chronic Obstructive Pulmonary Disease), ESRD (End Stage Renal Disease), HCV (Hepatitis C Virus) 


\begin{tabular}{|c|c|c|c|}
\hline Characteristics & $\begin{array}{l}\text { Readmitted } \\
(n=102)\end{array}$ & $\begin{array}{l}\text { Not Readmitted } \\
(n=338)\end{array}$ & $\begin{array}{c}P \text {-Value } \\
\text { Univariate analysis }\end{array}$ \\
\hline Age, in years, mean (SD) & $49.3(12.4)$ & $48.4(12.6)$ & 0.492 \\
\hline Male/Female & $64(62.7 \%) / 38(37.3 \%)$ & $183(54.1 \%) / 155(45.9 \%)$ & 0.125 \\
\hline Malignancy & $31(30.4 \%)$ & $55(16.3 \%)$ & $0.02^{*}$ \\
\hline VL>200 copies/ml & $47(46.1 \%)$ & $89(26.3 \%)$ & $<0.05^{*}$ \\
\hline CD4<200 cells $/ \mathrm{mm} 3$ & $43(42.2 \%)$ & $99(29.3 \%)$ & $0.015^{*}$ \\
\hline \multicolumn{4}{|l|}{ HIV-1 Genotype } \\
\hline GT-without drug resistance $(n=123)$ & $28(22.8 \%)$ & $95(77.2 \%)$ & 0.11 \\
\hline $\begin{array}{l}\text { GT-with drug } \\
\text { resistance }(n=112)\end{array}$ & $36(32.1 \%)$ & $76(67.9 \%)$ & 0.11 \\
\hline Final Model Variable & Odds Ratio & 95\% Confidence Interval & $\begin{array}{c}P \text {-Value } \\
\text { Multivariate analysis }\end{array}$ \\
\hline VL>200 copies/ml & 2.12 & $1.07-4.21$ & $0.032^{*}$ \\
\hline CD4<200 cells $/ \mathrm{mm} 3$ & 1.36 & $0.70-2.65$ & 0.363 \\
\hline Malignancy & 2.25 & $1.24-4.11$ & $0.008^{* *}$ \\
\hline
\end{tabular}

VL (viral load), GT (genotype), ${ }^{*}<0.05,{ }^{* *}<0.01$

Table 1: Patient characteristics and associations with re-admission.

comorbidity rates for DM-II of $11.8 \%(n=12)$, HTN of $38.2 \%(n=39)$, COPD of $12.7 \%(n=13)$, ESRD of $9.8 \%(n=10)$, HCV of $18.6 \%(n=19)$, malignancy of $30.4 \%(n=31)$ and psychiatric diagnosis of $36.3 \%(n=37)$ (Table 1).

During this 12 month period, 102 patients were readmitted within 30 days. Based on univariate analysis, age was not significantly associated with readmission $(\mathrm{p}=0.492)$, nor was male or female sex $(\mathrm{p}=0.125)$. Of those readmitted within 30 days, $30.4 \%(\mathrm{n}=31)$ had a comorbid diagnosis of malignancy while only $19.5 \%(n=86)$ of all index admissions had a co-morbid diagnosis of malignancy. Of the patients readmitted within 30 days, $46.1 \%(\mathrm{n}=47)$ had an elevated viral load (VL) $>200$ copies/ $\mathrm{ml}$ while only $26.3 \%(\mathrm{n}=89)$ of those not being readmitted within 30 days had an elevated VL $(\mathrm{p}<0.05)$. Of the patients readmitted within 30 days who reported compliance with HAART only $10.7 \%$ had an elevated viral load while $100 \%$ of those reporting noncompliance had an elevated viral load. Of the patients readmitted within 30 days, $42.2 \%(\mathrm{n}=43)$ had a CD4 count $<200$ cells $/ \mathrm{mm}^{3}$ while $29.3 \%(\mathrm{n}=99)$ of those not being readmitted within 30 days did $(\mathrm{p}=0.015)$ (Table 1$)$.

Genotype information was available in 235 of the 440 patients. This included baseline as well as any repeat genotypes obtained. Of the 123 patients with wild type HIV-1, 22.8\% ( $\mathrm{n}=28)$ were readmitted within 30 days ( $\mathrm{p}=0.11$ ) while of the 112 patients with resistance mutations detected, $32.1 \%(\mathrm{n}=36)$ were readmitted within 30 days $(\mathrm{p}=0.11)$ (Table 1$)$.

From this initial univariate analysis three variables were associated with increased rates of 30-day readmission; VL $>200$ copies $/ \mathrm{ml}$, CD4 count $<200$ cells $/ \mu \mathrm{L}$, and a co-morbid diagnosis of malignancy. Multivariable logistic regression determined the OR for readmission with $\mathrm{VL}<200$ copies/ml was 2.12 with a $95 \%$ CI of $1.07-4.21$ ( -value $=0.032$ ). The odds ratio (OR) for readmission with CD4 count $<200$ cells $/ \mathrm{mm}^{3}$ was 1.36 with a $95 \% \mathrm{CI}$ of $0.07-2.65$ (p-value $=0.363$ ). The OR for 30 -day readmission with a co-morbid diagnosis of malignancy was 2.25 with a 95\% CI of 1.24-4.11 (p-value $=0.008)$ (Table 1$)$.

The malignancies seen among the 86 index admissions were most commonly lymphomas $41 \%(\mathrm{n}=35)$ followed by lung cancer $12.8 \%$ $(\mathrm{n}=11)$ then KS $8.1 \%(\mathrm{n}=7)$. Malignancies in the patients re-admitted within 30 days were also most commonly lymphomas $38 \%(n=17)$ followed by lung cancer $20 \%(n=9)$ then KS $11 \%(n=5)$.

\section{Discussion}

Hospital readmissions within 30 days have an impact in terms of patient morbidity as well as financially, with hospitalizations for HIV1 -infected patients estimated to cost in excess of $\$ 15,000$ per admission $[12,13]$. Using this metric of $\$ 15,000$ per admission, the estimated cost of 30-day-readmissions in this cohort over these 2 years would be $>\$ 1.5$ million. In addition to costs, 30-day-readmission rates are publically reported and endorsed for inclusion in hospital quality profiling and pay-for-performance models and considered to be mostly preventable [14]. In our HIV-1-infected cohort thirty-day readmission rate for all patients was $23.2 \%$. This is in excess of Medicare rates of $18-19 \%$, and very similar to the estimated $24 \%$ readmission rate seen for patients discharged after an index admission for congestive heart failure $[15,16]$.

Our findings of increased readmission rates with poorly controlled HIV-1 viral loads and advanced HIV-1 with decreased CD4 counts confirm results of earlier studies [3]. This association with poor virological control directly correlates with poor compliance with medication and is consistent with prior investigations that have demonstrated a relationship between not taking HAART and readmission rates [5].

Our findings confirm earlier studies demonstrating poor control of HIV-1 and advanced AIDS as significant predictors of 30-day hospital readmissions. In our studied cohort, a comorbidity of malignancy was the most significant predictor of increased 30-day readmission rates. Of the 86 patients that met criteria for an index admission and had a comorbidity of malignancy, $36 \%$ of these patients were readmitted within 30 days. Patients with a comorbidity of malignancy made up $>30 \%$ of all patients readmitted within 30 days. Lymphoma was the most commonly seen malignancy in these patients, consistent with the literature in terms of incidence and deaths in HIV-1-infected patients $[9,10]$.

Important to our study was the availability of access to primary care for this cohort as inability to access outpatient care is associated with hospital readmissions and would have been a potentially confounding variable for our investigation. Our clinic is a funded Center for Clinical AIDS Research and Treatment (CART) and provides access to primary care, social work, mental health care, home base nursing 
when necessary and transportation vouchers if required. The fact that we studied a cohort with access to primary care with all these available support services is also a limitation for our study as our findings may not be directly applicable to populations without these services. It is reasonable to expect that in areas with poor access to physicians with expertise in HIV management this lack of access may impact 30-day readmission rates. A lack of social services and mental health services may also have a significant impact on readmissions. With availability for this cohort to be managed by home based nursing services many potential readmissions may have been prevented. Due to limitations our findings are not directly translatable to all HIV-1-infected patients.

Although not a primary focus of our study, we did observe that among patients with CD4 counts $<200$ cells $/ \mathrm{mm}^{3}$ infections as well as patients with uncontrolled VL, both AIDS-related and non-AIDSrelated were increased compared to patients with CD4 counts $>200$ cells $/ \mathrm{mm}^{3}$ and undetectable viral loads.

In summary, we found in a retrospective cohort study that the presence of malignancy as a comorbidity was a significant predictor of 30-day hospital readmission in HIV-1-infected patients. If our findings are reproduced at other centers in a prospective manner this would assist in evidence based cost effective utilization of resources and attention to this high-risk population for 30-day-hospital readmission [17].

\section{Authorship Contribution}

JCR participated in design of this project, classified admission diagnosis, analyzed data, and was involved in writing the manuscript and constructing figures. RMS analyzed data, ran statistical evaluations, and was involved in writing the manuscript. JM participated in design of this project, data analysis and was involved in writing the manuscript, DOG was involved in the design of the project, analyzed data and was involved in writing the manuscript and constructing figures.

\section{Funding Sources}

Donald and Barbara Zucker Family Foundation.

\section{References}

1. Berry SA, Fleishman JA, Moore RD, Gebo KA (2015) Thirty-day hospital readmissions for adults with and without HIV infection. HIV Med.

2. Nijhawan AE, Clark C, Kaplan R, Moore B, Halm EA, et al. (2012) An electronic medical record-based model to predict 30-day risk of readmission and death among HIV-infected inpatients. J Acquir Immune Defic Syndr 61: 349-358.
3. Berry SA, Fleishman JA, Yehia BR, Korthuis PT, Agwu AL, et al. (2013) Thirtyday hospital readmission rate among adults living with HIV. AIDS 27: 20592068.

4. Berry SA, Manabe YC, Moore RD, Gebo KA (2010) Hospitalization risk following initiation of highly active antiretroviral therapy. HIV Med 11: 289-298.

5. Nosyk B, Sun H, Li X, Palepu A, Anis AH (2006) Highly active antiretroviral therapy and hospital readmission: comparison of a matched cohort. BMC Infect Dis 6: 146.

6. Palepu A, Sun $H$, Kuyper L, Schechter MT, O'Shaughnessy MV, et al. (2003) Predictors of early hospital readmission in HIV-infected patients with pneumonia. J Gen Intern Med 18: 242-247.

7. Grant RW, Charlebois ED, Wachter RM (1999) Risk factors for early hospital readmission in patients with AIDS and pneumonia. J Gen Intern Med 14: 531536.

8. Griffin DO, Dharsee A, Rico JC, McGowan J (2014) Increasing Deaths Due to Malignancy in HIV+ Patients is Associated with Integrase Inhibitor Therapy. Journal of Health Science 2: 240-247.

9. Shiels MS, Pfeiffer RM, Gail MH, Hall HI, Li J, et al. (2011) Cancer burden in the HIV-infected population in the United States. J Natl Cancer Inst 103: 753-762.

10. Robbins HA, Pfeiffer RM, Shiels MS, Li J, Hall HI, et al. (2015) Excess cancers among HIV-infected people in the United States. J Natl Cancer Inst 107.

11. Griffin DO, Metzger M, Poeth K, Deng K, Dharsee A, et al. (2015) Malignancies, Particularly B-Cell Lymphomas, Are a Frequent Cause of Mortality in Human Immunodeficiency Virus-1 Patients Despite Highly Active Antiretroviral Therapy. Open Forum Infect Dis 2: ofv147.

12. Hellinger FJ (2007) The changing pattern of hospital care for persons living with HIV: 2000 through 2004. J Acquir Immune Defic Syndr 45: 239-246.

13. Hellinger FJ (2004) HIV patients in the HCUP database: a study of hospital utilization and costs. Inquiry 41: 95-105.

14. Kocher RP, Adashi EY (2011) Hospital readmissions and the Affordable Care Act: paying for coordinated quality care. JAMA 306: 1794-1795.

15. Krumholz HM, Merrill AR, Schone EM, Schreiner GC, Chen J, et al. (2009) Patterns of hospital performance in acute myocardial infarction and heart failure 30-day mortality and readmission. Circ Cardiovasc Qual Outcomes 2: 407-413.

16. Gerhardt G, Yemane A, Hickman P, Oelschlaeger A, Rollins E, et al. (2013) Medicare readmission rates showed meaningful decline in 2012. Medicare Medicaid Res Rev 3

17. Hellinger FJ (2015) Measuring the cost effectiveness of HIV prevention interventions in the US: pitfalls and problems. Appl Health Econ Health Policy 13: $129-133$. 